\title{
Seminal reactive oxygen species and total antioxidant capacity: Correlations with sperm parameters and impact on male infertility
}

\author{
Vidyalakshmi Subramanian 1, Aishwarya Ravichandran ', Nivethitha Thiagarajan', Matheswari Govindarajan', \\ Silambuchelvi Dhandayuthapani ${ }^{2}$, Sujatha Suresh ${ }^{2}$ \\ 'Department of Biotechnology, PSG College of Technology, Coimbatore; ${ }^{2}$ Vamsam Fertility Center, Coimbatore, India
}

Objective: The aim of this study was to measure reactive oxygen species (ROS) production and total antioxidant capacity (TAC) in the seminal fluid of the male partners in couples undergoing intrauterine insemination and to evaluate correlations between these values and their semen parameters.

Methods: The study was conducted at Vamsam Fertility Center, Coimbatore, India and enrolled 110 male patients from whom semen samples were collected. ROS production was measured by a thiobarbituric acid reactive species assay, and TAC was measured by a 2,2-diphenyl-2-picrylhydrazyl free radical assay. The differences in the TAC and malondialdehyde (MDA) levels between the subfertile and fertile groups were analysed. Correlations between sperm parameters and TAC and MDA levels were statistically analysed, and cutoff values with respect to the controls were determined. All hypothesis tests used were two-tailed, with statistical significance assessed at the level of $p<0.05$.

Results: A total of 87 subfertile and 23 fertile men were included in the study. The mean MDA level was significantly higher in the subfertile subjects than in the fertile subjects, and the mean antioxidant level was significantly lower in the subfertile subjects than in the fertile subjects. Seminal MDA levels were negatively associated with sperm concentration, motility, and morphology, whereas the opposite was seen with TAC levels.

Conclusion: Measurements of seminal TAC and ROS are valuable for predicting semen quality, and hence predicting the outcomes of fertility treatment.

Keywords: Male infertility; Reactive oxygen species; Sperm count; Sperm morphology; Sperm motility

\section{Introduction}

Infertility has become a widespread problem in recent years. For the normal fertilization process to occur, a healthy, undamaged sperm cell is vital. Approximately one-third of infertility problems are

Received: Dec 22, 2017 · Revised: Feb 7, 2018 · Accepted: Mar 29, 2018 Corresponding author: Vidyalakshmi Subramanian

Department of Biotechnology, PSG College of Technology, Peelamedu, Coimbatore, Tamil Nadu 641004, India

Tel:+91-9894593378 Fax:+91-0422-2573833 E-mail:vids21@gmail.com

*This work was supported by PSG Institution, Coimbatore, India.

This is an Open Access article distributed under the terms of the Creative Commons Attribution Non-Commercial License (http://creativecommons.org/licenses/by-nc/4.0/) which permits unrestricted non-commercial use, distribution, and reproduction in any medium, provided the original work is properly cited. attributed to the male partner. In order to improve the fertility rate, potential causes of damage leading to abnormal semen parameters should be evaluated. Semen analysis constitutes the initial step in screening for the male partner's contribution in an infertile couple. Extensive debate exists concerning the diagnostic and prognostic significance of semen parameters in the evaluation of male infertility, except in patients with azoospermia or severe oligozoospermia [1,2]. In couples experiencing idiopathic or unexplained infertility, routine semen analysis results in no detectable abnormalities.

Oxidative stress has been found to be one of the most prevalent causes of sperm damage, subsequently affecting fertilization [3]. Oxidative stress is a condition associated with an increased rate of sperm damage due to cellular damage to the lipids, DNA, and protein of the 
sperm $[4,5]$. Sperm cells have their own defence mechanisms to repair such damage, and when these fail, an imbalance between reactive oxygen species (ROS) and total antioxidant capacity (TAC) occurs [5]. This has been reported to be one of the most important factors contributing to poor semen quality. Low levels of ROS are important for capacitation and several other cell signalling pathways [6]. Elevated ROS levels, in contrast, cause a cascade of events leading to lipid peroxidation [5]. This results in an increase in the level of free radicals beyond the antioxidant capacity of the seminal plasma. Antioxidant levels are highly variable across men, but during fertility treatment, seminal antioxidant levels are rarely assessed in the male partner. After an assessment of routine male and female parameters, patients are usually counselled to proceed to intracytoplasmic sperm injection or intrauterine insemination [7]. One of the best strategies to enhance the potential for fertilization is to reduce the level of ROS in the seminal plasma by oral supplementation with antioxidants. This may increase the likelihood of natural conception, thereby improving the outcome of assisted reproductive technologies [8].

The purpose of this study was to quantify malondialdehyde (MDA), one of the by-products of lipid peroxidation and antioxidants in the seminal plasma. Measuring the level of free radicals and total antioxidants present in the seminal plasma enables an assessment of the correlations of these values with semen parameters such as motility, concentration, and morphology, thereby helping to improve the success rate of fertility treatment. Supplementing patients with necessary antioxidants for a prescribed duration after performing the antioxidant assay may help them to improve their antioxidant capacity and maintain an appropriate balance between ROS and antioxidants. This, in turn, may help maintain the integrity of DNA, leading to an improved fertility rate in patients with male factor infertility. Hence, standardizing these assays for measuring the level of total antioxidants present in the seminal plasma and establishing cutoff values for easy reference will be useful for treating patients by targeting oxidative stress.

\section{Methods}

\section{Study population}

The study was approved by the Institutional Human Ethics Committee (No. 15/278). Written informed consent was obtained from all subjects whose samples were analysed in this study. A questionnaire about the participants' lifestyle and medical history was also administered. Semen samples from 87 subfertile men attending a private fertility center in Coimbatore from May 2015 to April 2016 were obtained for this analysis. Twenty-three fertile men were enrolled as controls. The mean age of the fertile subjects and subfertile subjects was $33.73 \pm 4.64$ and $33.74 \pm 5.21$ years, respectively.
The study group included 26 normozoospermic male partners, 51 teratozoospermic male partners, 10 oligoteratozoospermic male partners, and 23 fertile healthy volunteers as a control group. The semen parameters were used to classify the patients into these groups following the 2010 World Health Organization (WHO) guidelines [9]. Semen samples were collected by masturbation after 3-4 days (7296 hours) of sexual abstinence. After liquefaction, semen analysis was performed according to the 2010 WHO guidelines within 1 hour of collection to measure sperm concentration, motility, and morphology [9]. Liquefied semen was centrifuged at $300 \times g$ for 10 minutes, and the sperm pellet was washed and resuspended in phosphatebuffered saline. The seminal plasma (supernatant) was carefully removed and transferred to microfuge tubes. Aliquots of seminal plasma were frozen at $-20^{\circ} \mathrm{C}$ until examination.

Subjects whose partners did not conceive after a period of unprotected intercourse for more than a year were included in the study, regardless of their semen parameters. Exclusion criteria are: (1) subjects who smoked and/or consumed alcohol, (2) subjects with leukocytospermia or pyospermia, (3) subjects suffering from diabetes mellitus or tuberculosis, or who had a previous history of mumps, and (4) subjects with a known male factor anomaly, such as varicocele.

\section{Thiobarbituric acid reactive species assay}

ROS levels were measured using a thiobarbituric acid reactive species assay [10]. This assay measured the level of MDA, which is one of the end products of lipid peroxidation. A $0.5-\mathrm{mL}$ aliquot of seminal plasma was mixed with $3 \mathrm{~mL}$ of $1 \%$ phosphoric acid and $1 \mathrm{~mL}$ of $0.6 \%$ thiobarbituric acid. The mixture was heated for 45 minutes in a water bath. Then, $4 \mathrm{~mL}$ of $n$-butanol was added and the mixture was vigorously vortexed and centrifuged at $300 \times g$ for 20 minutes. The upper organic layer was taken and absorbance was recorded at 535 $\mathrm{nm}$ in a ultraviolet-visible spectrophotometer. The values were expressed as $\mathrm{nmol} / \mathrm{mL}$. The extent of radical scavenging was calculated using the following formula:

$$
\frac{\text { Optical density } \times \text { total volume }}{1.56 \times 10^{5} \times \text { Sample volume }} \times 10^{6} \mathrm{nmol} / \mathrm{mL}
$$

\section{2,2-Diphenyl-2-picrylhydrazyl free radical assay}

TAC was measured using a 2,2-diphenyl-2-picrylhydrazyl free radical (DPPH) assay [11], in which $100 \mu \mathrm{L}$ of seminal plasma was added to $2.5 \mathrm{~mL}$ of DPPH solution. Then, another $2.4 \mathrm{~mL}$ of methanol was added, and the reaction mixture was allowed to stand in the dark at room temperature for 30 minutes. Methanol served as a blank, and DPPH in methanol without the sample served as a positive control. Following incubation, the discolouration of the purple colour was read at $515 \mathrm{~nm}$ in a spectrophotometer. The values were expressed as percentages. The extent of radical scavenging was calculated us- 
ing the following formula, where $\mathrm{Abs}_{\text {control }}$ and $\mathrm{Abs}_{\text {sample }}$ are the absorbance of the control and sample, respectively:

$$
\text { Percentage effect }(E \%)=\frac{A b s_{\text {control }}-A b_{\text {sample }}}{A b s_{\text {control }}} \times 100
$$

\section{Statistical analysis}

Data were expressed as mean \pm standard deviation. The MannWhitney $U$-test was used to assess differences between the subfertile and fertile groups. Spearman correlation analysis was used to calculate the coefficient of correlation. All hypothesis tests used were twotailed, and $p$-values $<0.05$ were considered to indicate statistical significance. A receiver operating characteristic $(\mathrm{ROC})$ curve was generated to determine the cutoff values with $95 \%$ confidence intervals. All statistical tests were performed using IBM SPSS ver. 19.0 (IBM Corp., Armonk, NY, USA).

\section{Results}

Semen samples from 110 individuals, including 87 subfertile partic- ipants (26 normozoospermic, 51 teratozoospermic, and 10 oligoteratozoospermic) and 23 fertile donors, were studied. The mean age of the fertile subjects and subfertile subjects was $33.73 \pm 4.64$ years and $33.74 \pm 5.21$ years, respectively. The semen parameters of both groups, including sperm concentration, motility, morphology, MDA levels, and TAC are presented in Table 1. The mean MDA level was significantly higher in the subfertile subjects $(15.31 \pm 12.03 \mathrm{nmol} / \mathrm{mL})$ than in the fertile subjects $(6.10 \pm 4.94 \mathrm{nmol} / \mathrm{mL})$. The mean antioxidant level was significantly lower in the subfertile subjects $(70.07 \% \pm 18.15 \%)$ than in the fertile subjects $(83.05 \% \pm 8.34 \%)$.

The correlations between semen parameters and seminal plasma ROS and TAC levels were examined using Spearman correlation analysis, as shown in Table 2. Unlike sperm morphology, a significant negative correlation was observed between the seminal MDA level and the percentage of sperm motility. The corresponding correlation for sperm concentration was close to significant. The presence of a negative correlation between seminal MDA levels and semen parameters meant that higher levels of MDA in the seminal plasma were associated with higher levels of abnormalities in the semen parameters. The

Table 1. Comparison of semen parameters, including ROS levels and TAC, between subfertile men and fertile controls

\begin{tabular}{|c|c|c|c|c|c|}
\hline Group & Sperm concentration $\left(10^{6} / \mathrm{mL}\right)$ & Motility (\%) & Morphology (\%) & $\mathrm{MDA}(\mathrm{nmol} / \mathrm{mL})$ & TAC (\%) \\
\hline Fertile control $(n=23)$ & $66.83 \pm 20.5$ & $70.6 \pm 4.5$ & $5.09 \pm 1.4$ & $6.1 \pm 5$ & $83.05 \pm 8.3$ \\
\hline Normozoospermia $(n=26)$ & $66.9 \pm 10$ & $70.7 \pm 7$ & $4.5 \pm 1.2$ & $11.14 \pm 7^{\mathrm{a})}$ & $71.63 \pm 16^{a)}$ \\
\hline Teratozoospermia $(n=51)$ & $53.22 \pm 18^{\mathrm{a})}$ & $67.53 \pm 7^{\mathrm{a})}$ & $1.82 \pm 1^{\mathrm{a})}$ & $16.8 \pm 15^{a)}$ & $70.73 \pm 19^{a)}$ \\
\hline Oligoteratozoospermia $(n=10)$ & $8.75 \pm 3^{\mathrm{a})}$ & $59.7 \pm 13^{\text {a) }}$ & $0.5 \pm 0.5^{\mathrm{a})}$ & $18.6 \pm 3^{\mathrm{a})}$ & $62.7 \pm 21^{\mathrm{a})}$ \\
\hline
\end{tabular}

Values are presented as mean \pm standard deviation.

ROS, reactive oxygen species; TAC, total antioxidant capacity; MDA, malondialdehyde.

a) Indicates statistical significance at $p<0.05$.

Table 2. Correlation of semen parameters with MDA and TAC levels

\begin{tabular}{lccccc}
\hline \multirow{2}{*}{ Semen parameter } & \multicolumn{2}{c}{ MDA level } & & \multicolumn{2}{c}{ TAClevel } \\
\cline { 2 - 3 } \cline { 5 - 6 } & Coefficient of correlation & $p$-value & & Coefficient of correlation & $p$-value \\
Sperm concentration & -0.183 & 0.056 & 0.084 & 0.384 \\
Motility & $-0.316^{\mathrm{a})}$ & 0.001 & 0.038 & 0.691 \\
Morphology & -0.056 & 0.186 & 0.051 &
\end{tabular}

MDA, malondialdehyde; TAC, total antioxidant capacity.

a)Indicates statistical significance at $p<0.01$.

Table 3. Results of the ROC curve analysis for TAC levels of subfertile men versus fertile controls

\begin{tabular}{lccccc}
\hline Group & Area under the curve & p-value & Cutoff value (\%) & Sensitivity (\%) & Specificity (\%) \\
\hline Normozoospermia & 0.759 & 0.002 & 75 & 73 & 53 \\
Teratozoospermia & 0.708 & 0.004 & 77.45 & 69 & 54 \\
Oligoteratozoospermia & 0.769 & 0.008 & 76 & 73 & 7 \\
Control vs. all three subfertile groups & 0.732 & 0.001 & 77.4 & 69 & 58 \\
\hline
\end{tabular}

ROC, receiving operator characteristic; TAC, total antioxidant capacity.

a)Indicates statistical significance at $p<0.05$. 


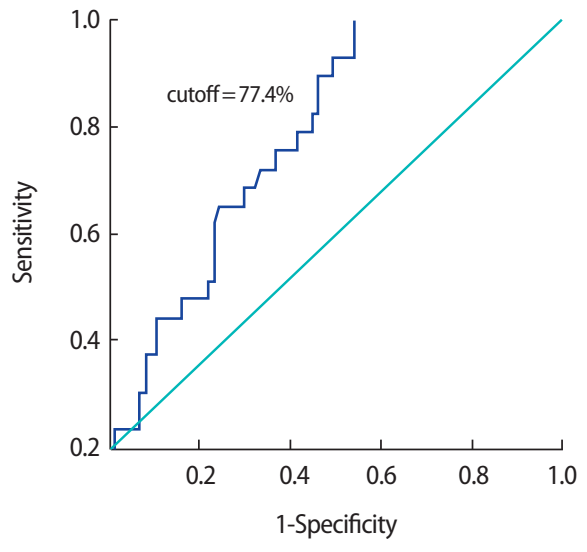

Figure 1. Receiver operating characteristic curve for total antioxidant capacity in subfertile men versus fertile controls. The area under the curve was 0.732 .

correlations between seminal TAC levels and sperm concentration, motility, and morphology were examined. Seminal TAC levels were positively correlated with sperm concentration, motility, and morphology, but the correlations were not statistically significant.

In order to analyze the effectiveness of oxidative stress in discriminating between the fertile and subfertile patients, an ROC curve was generated with a $95 \%$ confidence interval for TAC levels, comparing the fertile controls with the subfertile participants. The results are presented in Table 3.

Cutoff values for TAC were derived through a comparison of the control group with the individual subgroups of subfertile participants, as well as through a comparison of the control group with the subfertile participants as a whole. No effective cutoff value of TAC was derived when individual subgroups were compared with the fertile controls, while a TAC value of $77.4 \%$ was determined to be an efficient discriminating value when comparing the fertile participants with the subfertile participants as a whole (Figure 1). The low specificity $(7 \%)$ of oligoteratozoospermia may have been due to the very small sample size that was analysed.

\section{Discussion}

Imbalances between ROS and TAC in the male reproductive tract have a major influence on the fertilization potential of sperm. Measuring and standardizing these parameters would be helpful for treating patients with antioxidant therapy in order to increase the natural conception rate. The subfertile men included in this study had lower seminal TAC levels than the fertile men. Antioxidant levels showed positive associations with sperm concentration, motility, and morphology. The lower TAC levels in subfertile men were accompanied by higher levels of free radicals. The mean MDA level was signifi- cantly higher in the subfertile subjects than in the fertile subjects; this was due to the overproduction of ROS in semen, which is associated with reduced sperm fertilizing potential [12].

Based on the ROC curve, the most suitable cutoff value for TAC levels, along with sensitivity, specificity, and the area under the curve (AUC), was obtained by comparing the control group with the subgroups of subfertile men, as well as by comparing the control group with the subfertile participants as a single group. The cutoff value that was found is important because it discriminates between fertile and subfertile patients and provides a threshold value for various semen parameters based on clinical data. Previous studies have shown sperm morphology to have a high predictive power of various semen parameters, with AUCs of 0.78 and 0.697 , and the $4 \%$ cutoff value of sperm morphology showed good predictive value, as shown by an AUC of 0.782 [13]. Similarly, in our results, a significant TAC threshold value was found based on comparisons between the control and the subfertile subgroups and based on a comparison between the control group and the subfertile group as a whole. The threshold value of the TAC of semen that best discriminated between fertile and subfertile subjects was $77.4 \%$, with an AUC of 0.732 , a sensitivity of $69 \%$, and a specificity of $58 \%$, and we expect that these findings will have a clinical impact on screening for infertility treatment.

In addition to normal sperm concentration and other semen parameters measured in routine semen analysis, other markers are also needed to assess the fertilization potential [14]. Small amounts of ROS have been reported to be essential for the regulation of normal sperm function, but ROS have harmful effects beyond a certain level [15]. Oxidative stress develops as a result of imbalance between ROS generation and antioxidant scavenging. Morphologically abnormal spermatozoa and leukocytes are the main source of excess ROS generation in semen [15]. Activated leukocytes are capable of producing 100 -fold more ROS, than nonactivated leukocytes, so sperm DNA is more prone to leukocyte-induced ROS damage in infertile men and those with abnormal semen parameters because the sperm cells in such individuals are likely to have experienced DNA damage and have a more fragile chromatin structure.

At high levels of lipid peroxidation, spermatozoa are rendered dysfunctional and their membrane function is altered. Lipid peroxidation triggers the loss of membrane integrity, causing increased cell permeability, enzyme inactivation, structural damage to DNA, and cell death [15]. Increased formation of ROS has been associated with reduced sperm motility [16]; in accordance with that study, our study also showed a significant negative correlation between sperm motility and MDA levels. Previous studies have shown ROS to be negatively correlated with semen parameters [16,17]. Similarly, in our study, we found ROS to be negatively associated with sperm parameters such as sperm concentration, motility, and morphology. This associa- 
tion may be explained by decreased axonemal protein phosphorylation, which leads to sperm immobilization. Moreover, it has been hypothesized that $\mathrm{H}_{2} \mathrm{O}_{2}$ diffuses across the membrane and inhibits the activity of enzymes such as glucose-6-phosphate dehydrogenase, which in turn leads to reduced availability of the cytoplasmic enzyme $\mathrm{NADPH}$. As a result, oxidized glutathione and reduced glutathione accumulate, and these changes can cause a decrease in the antioxidant capacity of spermatozoa [18].

Initially, antioxidants are present in spermatozoa in a quantity capable of counteracting the free radicals that are produced such that a balance is maintained between antioxidants and ROS. On prolonged exposure, the antioxidant mechanism breaks down and an imbalance is created, due to which DNA fragmentation occurs [19]. Single-strand breaks are caused by oxidative stress. Double-strand breaks are caused due to exposure to 4-hydroxyl-2-nonenal, a major product of lipid peroxidation. The major DNA adducts found in human sperm DNA are 8-hydroxy-2-deoxyguanosine and 2-ethenonucleosides, which are used as key biomarkers for DNA damage caused by oxidative stress. Such DNA damage adversely affects the outcomes of assisted reproduction techniques and natural conception [19].

In accordance with previous studies in which morphologically abnormal spermatozoa have been suggested to be a potential source of ROS, in our study, participants with teratozoospermia and oligoteratozoospermia showed elevated MDA levels. Of the three subfertile groups included in the study, the patients with oligoteratozoospermia seem to have had significantly low TAC levels and very high MDA levels. In this condition, morphologically immature or abnormal sperm cells, which are a potential source of ROS, lead to increased lipid peroxidation. The decline in sperm concentration in these patients might have been due to extended exposure to high levels of ROS produced by immature or abnormal spermatozoa. This likely resulted in damage to tubules, causing testicular atrophy, reduction in motility, and DNA damage to mature sperm cells, all of which affect fertility potential.

Exogenous antioxidant supplementation should be considered as a therapeutic measure in patients with abnormal TAC and MDA levels. Several in vitro and in vivo studies have analysed the effects of different antioxidants on sperm parameters, such as vitamin $E$, vitamin $C$ (ascorbate), glutathione, and albumin. A dosage-dependent improvement in sperm quality was observed in smokers who took vita$\min$ C orally [20]. A randomized double-blind placebo-controlled trial reported that vitamin E supplementation significantly improved sperm motility in $60 \%$ of asthenozoospermic patients [21]. Supplementation with glutathione for 60 days in 10 infertile patients significantly reduced lipid peroxidation potential, and also led to improvement in sperm membrane characteristics [22]. A combination of an- tioxidants may be most useful in the treatment of male infertility in patients with elevated oxidative stress.

In conclusion, our study demonstrated that there were significant differences in the levels of ROS and TAC in the seminal plasma of subfertile and fertile participants. A significant threshold level for the TAC of semen (77.4\%) was established. Supplementation with necessary antioxidants for a certain period of time after performing an antioxidant assay may help patients to improve their antioxidant capacity and maintain a balance between ROS and antioxidants. This, in turn, may help maintain the integrity of DNA, thereby improving the fertility rate in patients with male factor infertility. Hence, standardizing these assays for measuring the total level of antioxidants present in the seminal plasma and establishing a cutoff value for easy reference will facilitate the treatment of patients for oxidative stress.

\section{Conflict of interest}

No potential conflict of interest relevant to this article was reported.

\section{Acknowledgments}

The authors are grateful to the PSG Institution, Coimbatore, India for funding this work.

\section{References}

1. Ombelet W, Bosmans E, Janssen M, Cox A, Vlasselaer J, Gyselaers $W$, et al. Semen parameters in a fertile versus subfertile population: a need for change in the interpretation of semen testing. Hum Reprod 1997;12:987-93.

2. Tomlinson MJ, Kessopoulou E, Barratt CL. The diagnostic and prognostic value of traditional semen parameters. J Androl 1999;20:588-93.

3. Aitken RJ, Smith TB, Jobling MS, Baker MA, De luliis GN. Oxidative stress and male reproductive health. Asian J Androl 2014;16:318.

4. Gavriliouk D, Aitken RJ. Damage to sperm DNA mediated by reactive oxygen species: its impact on human reproduction and the health trajectory of offspring. Adv Exp Med Biol 2015;868:23-47.

5. Aitken RJ, Gibb Z, Baker MA, Drevet J, Gharagozloo P. Causes and consequences of oxidative stress in spermatozoa. Reprod Fertil Dev 2016;28:1-10.

6. Aitken RJ. Reactive oxygen species as mediators of sperm capacitation and pathological damage. Mol Reprod Dev 2017;84:103952.

7. Tremellen K. Oxidative stress and male infertility: a clinical perspective. Hum Reprod Update 2008;14:243-58. 
8. Agarwal A, Sekhon LH. The role of antioxidant therapy in the treatment of male infertility. Hum Fertil (Camb) 2010;13:217-25.

9. World Health Organization. WHO laboratory manual for the examination and processing of human semen. 5th ed. Geneva: World Health Organization; 2010.

10. Wrolstad RE, Acree TE, Decker EA, Penner MH, Reid DS, Schwartz SJ, et al. Handbook of food analytical chemistry: water, proteins, enzymes, lipids, and carbohydrates. New Jersey: Wiley; 2005.

11. Garcia EJ, Oldoni TL, Alencar SM, Reis A, Loguercio AD, Grande $\mathrm{RH}$. Antioxidant activity by DPPH assay of potential solutions to be applied on bleached teeth. Braz Dent J 2012;23:22-7.

12. Cummins JM, Jequier AM, Kan R. Molecular biology of human male infertility: links with aging, mitochondrial genetics, and oxidative stress? Mol Reprod Dev 1994;37:345-62.

13. Menkveld R, Wong WY, Lombard CJ, Wetzels AM, Thomas CM, Merkus $\mathrm{HM}$, et al. Semen parameters, including $\mathrm{WHO}$ and strict criteria morphology, in a fertile and subfertile population: an effort towards standardization of in-vivo thresholds. Hum Reprod 2001;16:1165-71.

14. Agarwal A, Sharma RK, Nallella KP, Thomas AJ Jr, Alvarez JG, Sikka SC. Reactive oxygen species as an independent marker of male factor infertility. Fertil Steril 2006;86:878-85.

15. Griveau JF, Le Lannou D. Reactive oxygen species and human spermatozoa: physiology and pathology. Int J Androl 1997;20:619.
16. de Lamirande E, Gagnon C. Reactive oxygen species and human spermatozoa. II. Depletion of adenosine triphosphate plays an important role in the inhibition of sperm motility. J Androl 1992; 13:379-86.

17. Shamsi MB, Venkatesh S, Kumar R, Gupta NP, Malhotra N, Singh N, et al. Antioxidant levels in blood and seminal plasma and their impact on sperm parameters in infertile men. Indian J Biochem Biophys 2010;47:38-43.

18. Aitken RJ, Fisher HM, Fulton N, Gomez E, Knox W, Lewis B, et al. Reactive oxygen species generation by human spermatozoa is induced by exogenous NADPH and inhibited by the flavoprotein inhibitors diphenylene iodonium and quinacrine. Mol Reprod Dev 1997;47:468-82.

19. Gonzalez-Marin C, Gosalvez J, Roy R. Types, causes, detection and repair of DNA fragmentation in animal and human sperm cells. Int J Mol Sci 2012;13:14026-52.

20. Dawson EB, Harris WA, Rankin WE, Charpentier LA, McGanity WJ. Effect of ascorbic acid on male fertility. Ann N Y Acad Sci 1987; 498:312-23.

21. Suleiman SA, Ali ME, Zaki ZM, el-Malik EM, Nasr MA. Lipid peroxidation and human sperm motility: protective role of vitamin $\mathrm{E}$. J Androl 1996;17:530-7.

22. Lenzi A, Culasso F, Gandini L, Lombardo F, Dondero F. Placebocontrolled, double-blind, cross-over trial of glutathione therapy in male infertility. Hum Reprod 1993;8:1657-62. 